\title{
Editorial
}

\section{Function Spaces and Applications}

\author{
Henryk Hudzik, ${ }^{1}$ Vakhtang M. Kokilashvili, ${ }^{2}$ Baoxiang Wang, ${ }^{3}$ and Marek Wisła ${ }^{1}$ \\ ${ }^{1}$ Faculty of Mathematics and Computer Science, Adam Mickiewicz University in Poznań, Umultowska 87, 61-614 Poznań, Poland \\ ${ }^{2}$ Andrea Razmadze Mathematical Institute, Tamarashvili 6, 0177 Tbilisi, Georgia \\ ${ }^{3}$ LMAM, School of Mathematical Sciences, Peking University, Beijing 100871, China \\ Correspondence should be addressed to Henryk Hudzik; henryk.hudzik@amu.edu.pl
}

Received 3 July 2014; Accepted 3 July 2014

Copyright (C) 2015 Henryk Hudzik et al. This is an open access article distributed under the Creative Commons Attribution License, which permits unrestricted use, distribution, and reproduction in any medium, provided the original work is properly cited.

Function spaces and its various applications are subjects of investigation in many research centers and we are glad that this special issue has received the remarkable attention by researchers in these areas.

We have received papers from wide range of topics of function spaces such as interpolation methods for stochastic processes spaces, vector optimization problems in Banach spaces, Marcinkiewicz integral operator in Morrey spaces, Hardy's operator in the variable exponent Lebesgue spaces, and differential equations of weakly parabolic type in Banach spaces.

A number of papers are devoted to investigating different kinds of problems in Hardy spaces or Hardy subspaces, for example, Calderon-Zygumnd singular integral operators in Hardy spaces, weighted Hardy spaces related to the Schrödinger operator, and composition operator in hyperbolic Bloch-type spaces.

Geometric problems such as modulus of convexity in some Banach sequence spaces, $p$-uniform convexity, and $q$ uniform smoothness in $C^{2}$ spaces are also covered by this issue.

\section{Acknowledgment}

The editors thank all the contributors and colleagues who were prompt for referee work.

Henryk Hudzik Vakhtang M. Kokilashvili

Baoxiang Wang

Marek Wista 


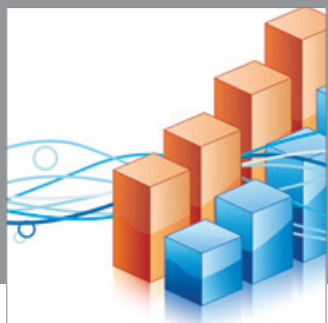

Advances in

Operations Research

mansans

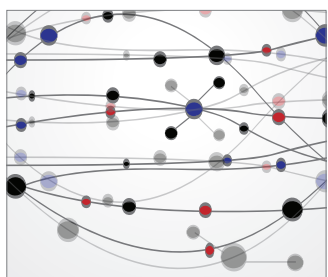

The Scientific World Journal
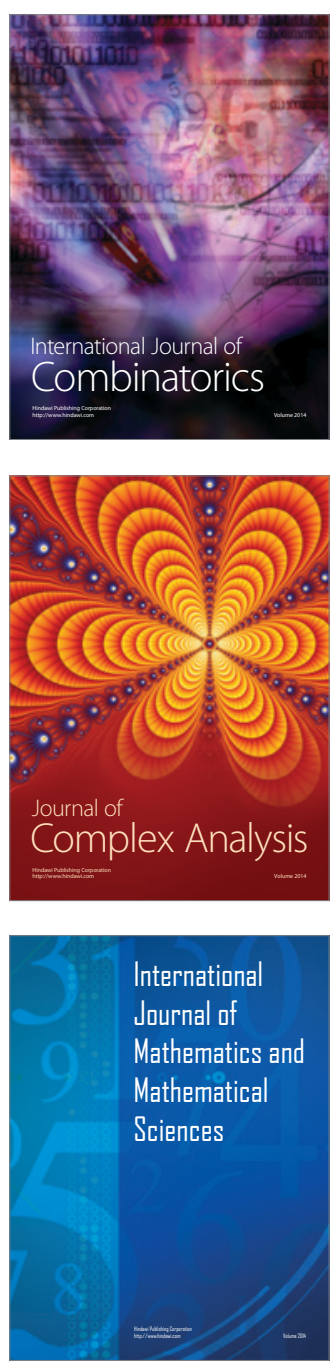
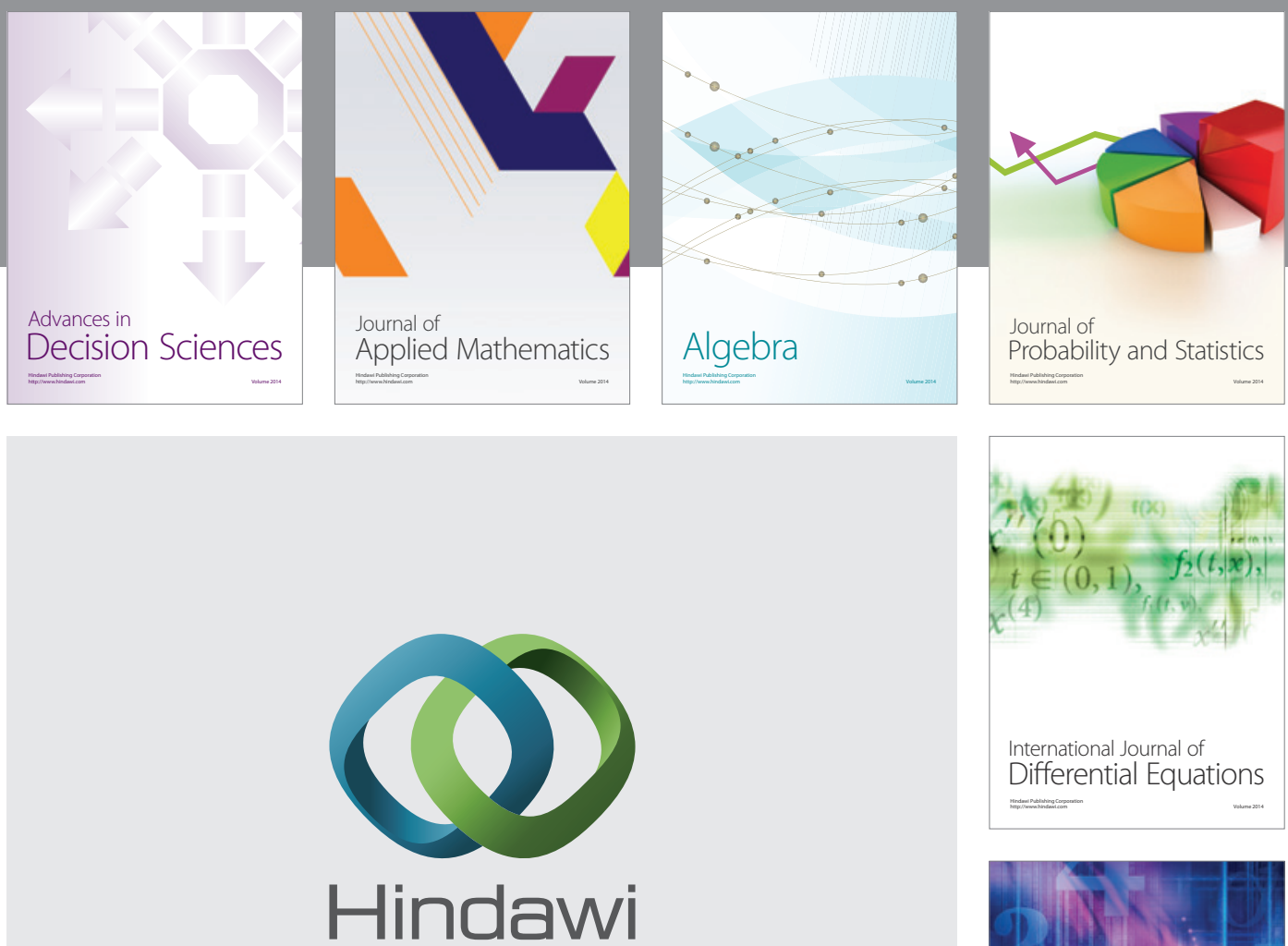

Submit your manuscripts at http://www.hindawi.com
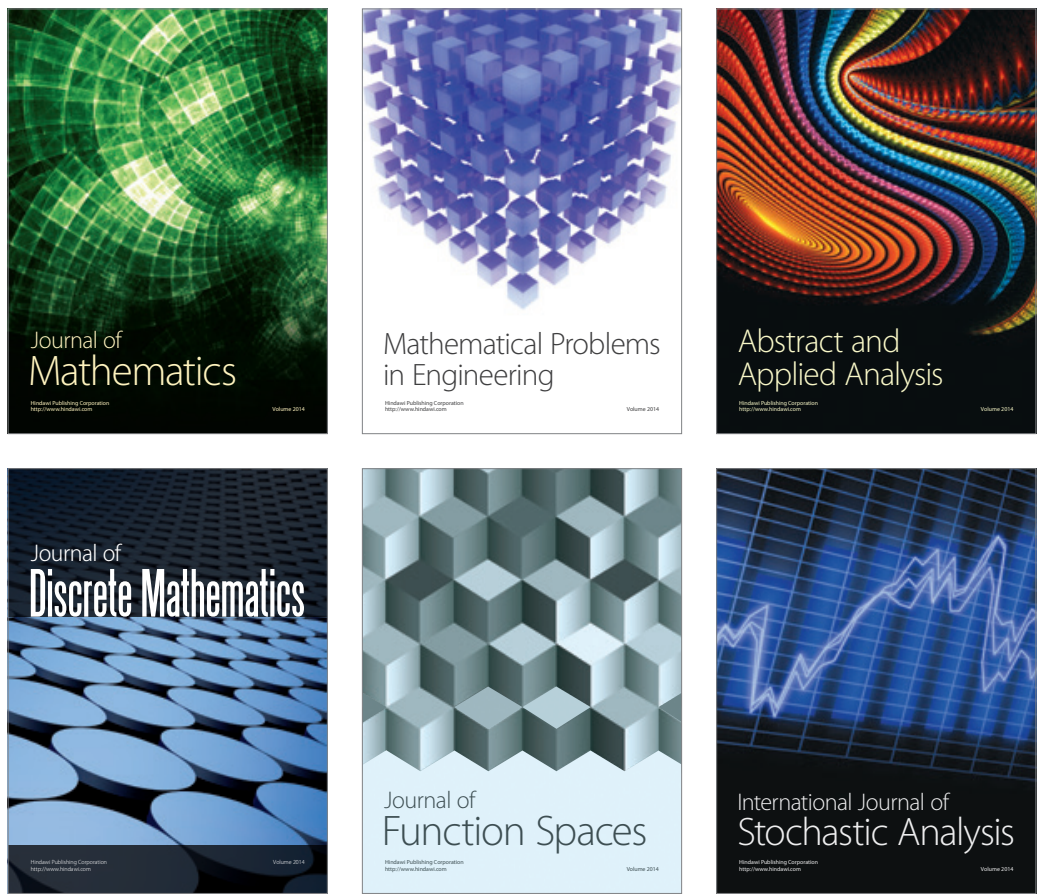

Journal of

Function Spaces

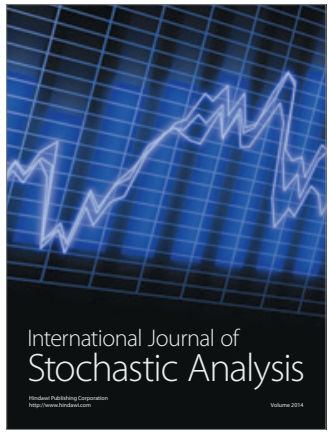

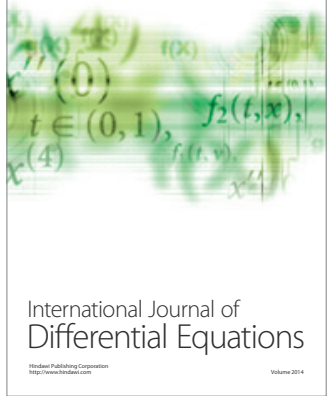
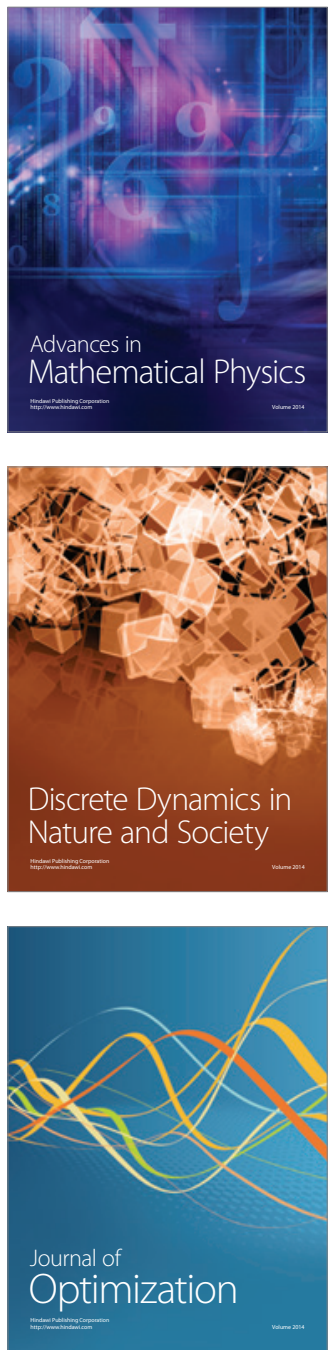